\title{
Ils ont leur mot à dire
}

Une école élémentaire à Boston, Massachusetts

Giving everyone a say - An elementary school in Boston (USA)

Tienen una voz. Una escuela primaria en Boston, Massachussetts

\section{Karen L. Mapp}

\section{(2) OpenEdition} Journals

Édition électronique

URL : https://journals.openedition.org/ries/1936

DOI : 10.4000/ries. 1936

ISSN : 2261-4265

\section{Éditeur}

France Education international

\section{Édition imprimée}

Date de publication : 1 décembre 2002

Pagination : 81-93

ISBN : 978-2854-2-0555-8

ISSN : $1254-4590$

\section{Référence électronique}

Karen L. Mapp, « Ils ont leur mot à dire », Revue internationale d'éducation de Sèvres [En ligne], 31 | décembre 2002, mis en ligne le 24 novembre 2011, consulté le 05 juillet 2021. URL : http:// journals.openedition.org/ries/1936; DOI : https://doi.org/10.4000/ries.1936 


\title{
Ils ont leur mot à dire
}

\section{Une école élémentaire à Boston, Massachusetts}

\author{
Karen L. Mapp
}

Ces dix dernières années, un regain d'intérêt a été observé au plan national pour le rôle que jouent les familles dans le développement éducatif de leurs enfants. Plus de trente années de recherche ont montré que, indépendamment du bagage économique, racial/ethnique ou éducatif, il existe un lien fort entre les différentes formes que peut recouvrir l'engagement des familles et le bénéfice qu'en tirent les enfants. Cet engagement comprend notamment l'encouragement à réussir à l'école, l'investissement dans des activités à la maison comme l'aide aux devoirs, le bénévolat à l'école et la participation aux activités d'administration de l'école.

Parmi les bénéfices que les enfants en tirent, on relève de meilleures notes et de meilleurs résultats aux tests, une plus grande assiduité aux cours, l'obtention de diplômes plus élevés, un nombre supérieur d'inscriptions dans l'enseignement post-secondaire et des attitudes plus positives à l'égard de l'école (Henderson \& Berla, 1994). En conséquence de ce lien entre l'engagement des familles et la réussite des élèves, plusieurs des projets actuels de réforme globale de l'école identifient le partenariat entre l'école, la famille et la communauté comme une composante des écoles à succès. A titre d'exemple, dans le programme pilote Comprehensive School Reform (Loi Obey-Porter, 1998) ${ }^{1}$, «la participation des parents et de la communauté» est l'un des huit pré-requis essentiels des stratégies développées par les écoles visant un haut niveau de qualification pour tous leurs élèves.

Cependant, malgré l'accent sur le partenariat entre écoles et familles, les professeurs, les directeurs et les parents qui essaient de coordonner les initiatives d'engagement des familles expriment leur frustration face au faible succès que rencontrent les programmes qui visent à tisser des liens significatifs et durables avec les familles. Dans certains cas, l'échec est imputé au manque d'implication des familles. Cette revendication d'une participation minimale est particulièrement répandue au sein des écoles qui accueillent les enfants des familles à faibles revenus et issues des minorités.

1. Le Comprehensive School Reform (CSR) program a pour objectif d'augmenter les performances des enfants, grâce à l'application de réformes globales fondées à la fois sur des recherches qui reposent sur des bases scientifiques solides, et des pratiques qui ont fait leurs preuves. Le public cible de ce programme sont les écoles connues pour leur pauvreté et enregistrant des résultats faibles. Pour plus d'information, consulter le site fédéral du ministère de l’Éducation : http://www.nochildleftbehind.gov/. 


\section{UNE ÉTUde de CAS}

Compte tenu du nouvel intérêt porté au rôle des familles dans le développement éducatif de leurs enfants, l'objectif de l'étude était d'identifier les facteurs de succès des partenariats éducatifs entre le personnel de l'école et les familles. La méthode retenue a consisté à demander aux parents ${ }^{2}$ comment et pourquoi ils étaient impliqués dans l'éducation de leurs enfants et quels étaient les facteurs qui influençaient leur participation. L'étude a été menée à l'école élémentaire Patrick O'Hearn à Boston, Massachusetts, où, d'après des renseignements collectés dans des enquêtes annuelles par l'école, au moins $90 \%$ des parents participaient à une ou plusieurs activités d'engagement des familles, qu'elles se déroulent à l'école ou à la maison. Cette école urbaine accueillait les enfants à partir de l'âge de cinq ans et fonctionnait selon le mode de full inclu$\operatorname{sion}^{3}$. Elle comptait 220 élèves dont approximativement $67 \%$ avaient droit à des repas gratuits ou à tarif réduit selon les ressources des familles. La population de l'école regroupait plusieurs races : $55 \%$ des élèves étaient afro-américains, $34 \%$ blancs, $6 \%$ hispaniques et $5 \%$ asiatiques. Un quart des 220 enfants était reconnu comme ayant des besoins spécifiques ${ }^{4}$. Entre 1989 et 1995, la moyenne des scores médians ${ }^{5}$ de l'école O'Hearn aux tests annuels de l'État du Massachusetts pour les classes 1 à $5^{6}$ a augmenté de $18 \%$ de points en anglais, (passant de 44 à 62), et de $31 \%$ de points en mathématiques (de 48 à 79).

Avec une majorité d'élèves ayant droit à des repas gratuits ou à un moindre coût, un taux de participation des parents de $90 \%$ et l'amélioration des résultats scolaires, l'école O'Hearn offrait un cadre favorable pour mener l'étude, c'est à dire pour demander aux parents aux bas revenus les raisons de leur implication dans l'éducation de leurs enfants et pour explorer les facteurs qui influencent leur participation.

Les parents des communautés urbaines de milieu socio-économique peu élevé ont souvent la réputation d'être difficiles à motiver. Cette recherche visait à comprendre en profondeur les perceptions que ces parents ont de leur participation à l'éducation de leurs enfants. Le manque d'implication de la part des parents aux revenus faibles est souvent attribué à leur «manque de temps, d'intérêt, de compétence ou de valorisation de l'éducation» (Davies, 1998, p. 53). Rassemblant les descriptions faites par les parents eux-mêmes de leur participation, l'étude cherchait à savoir elles allaient correspondre aux typolo-

2. Dans cette étude, le terme «parent» désigne tout adulte prenant en charge un enfant.

3. NdT : Le principe des full inclusion schools est d'éduquer les enfants ayant des difficultés avec ceux qui n'en ont pas, en les intégrant la plupart du temps dans une classe ordinaire, dans l'école de leur quartier.

4. L'expression "special needs» recouvre un ensemble de difficultés tant du domaine pédagogique ou socioculturel que médical qui nécessitent une prise en charge spécifique de l'enfant (NdIR).

5. La médiane est un indice statistique qui sépare la distribution des notes en deux parties égales. Pour chacun des cinq niveaux, on a relevé cet indice (NdIR).

6. NdT : Les classes 1 à 5 accueillent les enfants entre 6 et 10 ans. 
gies existantes, définies par Epstein (le statut de parent, la communication, le bénévolat, l'apprentissage à la maison, la prise de décision et l'implication visà-vis de la communauté; Epstein, 1988, 1991 et 1995) ou si elles échapperaient à cette catégorisation. Dans cette dernière hypothèse, il serait nécessaire de donner une définition élargie de l'implication des parents et/ou une définition plus globale sur le plan culturel.

L'étude visait aussi à rechercher les facteurs influents en essayant de comprendre les motivations, les attentes et les perceptions qui jouent un rôle dans la participation des parents au développement éducatif de leurs enfants et/ou dans les programmes d'implication des familles.

\section{L'ÉCOLE O'HEARn \\ ET L'ÉVOLUTION DE SON PROGRAMME D'IMPLICATION DES FAMILLES}

Au début de l'année 1989, l'école élémentaire Patrick O’Hearn ${ }^{7}$ fut choisie pour être la première école de Boston à accueillir un programme de full inclusion à partir de l'âge de cinq ans. Durant l'été 1989, William Henderson fut nommé directeur de l'école. A la rentrée scolaire, Henderson chargea l'équipe d'enseignants d'une étude dont l'objectif était d'identifier les thèmes considérés comme les priorités absolues de l'école. Les résultats de l'étude indiquaient de manière écrasante le besoin d'une plus grande implication des familles. Les enseignants d'O'Hearn désignèrent alors un groupe d'une dizaine de parents, qui formèrent le comité de participation des familles d'O'Hearn. Ce comité était hétérogène d'un point de vue ethnique et socio-économique et présentait également des différences quant à la composition des familles : en étaient membres des familles monoparentales, des grands-parents et des parents dont les enfants avaient ou non des besoins spécifiques.

\section{La participation des parents à l'administration de l'école}

Outre la formation d'un comité de participation des familles, les parents, soutenus par Henderson, commencèrent à s'engager activement dans l'administration de l'école. En 1990, une mesure prit effet dans le district, autorisant les écoles de Boston à adopter sur la base du volontariat des structures administratives appelées conseils d'école ${ }^{8}$. Sur la demande de l'équipe de

7. Par la suite, «O’Hearn» désignera l'école élémentaire Patrick O’Hearn.

8. En 1990, le district scolaire de Boston adopta une mesure sur les conseils d'école, qui devenaient partie prenante de l'administration des écoles. En 1993, la mesure devint obligatoire pour l'ensemble du district. Les conseils se composaient du principal et d'un nombre équivalent d'enseignants et de parents. Ils devaient refléter l'équilibre ethnique de la population de l'école qu'ils représentaient. 
renforcer l'implication des familles dans l'école, Henderson encouragea l'équipe enseignante et les membres du comité de participation des familles à constituer un tel conseil. Les enseignants et les parents acceptèrent et en 1990 O'Hearn fut l'une des 35 écoles sur les 117 que comptait le district à adopter bénévolement le système d'administration fondé sur le conseil d'école. Un groupe constitué du directeur, de sept parents et de sept enseignants d'origines ethniques diverses, commença à se réunir tous les mois pour réfléchir en priorité aux questions d'enseignement et d'apprentissage à l'école.

\section{La formation du Family Outreach Program 9}

A l'automne 1991, O’Hearn reçut une subvention de l'Institute for Responsive Education afin de soutenir les efforts déployés par l'école pour mettre en valeur les programmes de partenariat entre l'école, les familles et les communautés. La subvention exigeait de l'école qu'elle forme une équipe composée de parents, d'enseignants et du principal, à qui était confiée la responsabilité d'étendre et de renforcer le programme de participation de familles d'O'Hearn. Suite à cette subvention, le family outreach team d'O'Hearn vit le jour, composé de nouveaux parents recrutés pour rejoindre l'équipe, de membres de l'ancien comité pour la participation des familles, d'un professeur qui s'était proposé pour travailler pour le comité et du directeur (Palanki \& Burch, 1995). Le groupe était toujours homogène, avec une quinzaine de parents et de grandsparents bénévoles de milieux afro-américains, blancs et latinos et un mélange de parents dont les enfants avaient des besoins spécifiques et d'autres pas. La subvention permettait aussi de bénéficier des services d'un «facilitateur» qui travaillait avec le groupe une journée par semaine.

La nouvelle outreach team d'O'Hearn cherchait en particulier à développer des relations avec les parents qui n'avaient que rarement, voire jamais, de contacts avec l'école. L'outreach team décida que le face à face, le contact de parents à parents, était une manière d'esquisser une relation entre les familles et l'école. Au titre de l'outreach strategy, l'équipe développa un programme de visites à domicile. Celles-ci ne cherchaient pas à donner un cours aux parents sur la manière dont ils devaient participer à l'éducation de leurs enfants, mais servaient à transmettre le message que les familles étaient respectées et accueillies au sein de la communauté d'O'Hearn.

\section{L'ouverture du centre familial}

En travaillant en collaboration avec Henderson et les enseignants, le comité des parents réunit des fonds et organisa le futur centre familial à l'inté-

9. Programme destiné à informer les familles défavorisées de leurs droits et des programmes d'assistance dont elles peuvent bénéficier. 
rieur de la bibliothèque de l'école. Au printemps 1991, le O'Hearn Family Center fut inauguré. Ayant pour vocation de faire en sorte que les familles se sentent accueillies par l'école, il offrait un cadre confortable pour que les familles se rassemblent autour d'un rafraîchissement et discutent de façon informelle de sujets sociaux et éducatifs. Avec le temps, le Centre fut régulièrement utilisé à la fois par les parents et par les professeurs pour des événements tels que des petits-déjeuners, des occasions particulières, des conférences parents/enseignants, ainsi que des activités d'accueil des familles.

\section{Autres composantes du programme de participation des familles}

Le travail de l'outreach team donna naissance à plusieurs autres projets d'implication familiale, comme la publication mensuelle d'une lettre d'information de l'école et la création de la O'Hearn family leadership team, qui comptait également parmi ses membres des enseignants. Ce groupe se rassemblait chaque mois pour poursuivre le travail de promotion d'un environnement qui accueille l'ensemble des familles d'O'Hearn et, selon le principe de full inclusion, accepte tous les enfants.

Les membres de l'outreach team organisaient en outre des ateliers afin d'améliorer leurs propres compétences en tant que volontaires de l'Outreach Program. En collaborant avec l'équipe d'O'Hearn, ils développèrent des nouvelles stratégies pour encourager les familles à venir à l'école, pour les y accueillir, leur donner des renseignement d'ordre éducatif et pour leur proposer de l'aide.

\section{PrincipauX RÉsultats DE L'ÉTUdE}

Les réponses à la première question «pourquoi et comment les parents ayant de faibles revenus sont engagés dans l'éducation de leurs enfants?» ont fait apparaître trois thèmes.

Les parents interviewés confirmaient fortement la thèse de la recherche, selon laquelle la majorité des parents, quels que soient leur race, leur ethnie ou leur statut socio-économique, sont vivement intéressés par l'éducation de leurs enfants. Ils exprimaient un désir authentique et profond d'aider leurs enfants à réussir à l'école et se montraient motivés pour faire tout leur possible pour assurer le succès scolaire de leurs enfants. Leurs témoignages remettaient ainsi en cause l'idée selon laquelle les parents aux revenus faibles «n'accordent aucune importance » à l'éducation de leurs enfants.

Les parents comprenaient en outre clairement que leur engagement aidait le développement éducatif de leurs enfants. Ils disaient avoir perçu des 
différences dans le comportement et les sentiments de leurs enfants par rapport à l'école, depuis qu'eux-mêmes s'engageaient à la maison et/ou à l'école. Ils y voyaient un rapport de cause à effet. Les réactions positives des enfants étaient un facteur de motivation pour assurer dans la durée le soutien des parents.

Enfin, l'engagement des parents pouvait prendre différentes formes qui n'étaient pas toutes reconnues par l'équipe de l'école, dont certains membres avaient une vision étroite de ce qui peut constituer une participation légitime. L'engagement des parents regroupait un large éventail d'activités qui se déroulaient à la fois à la maison et à l'école. Outre des formes plus traditionnelles d'engagement, les parents apportaient un soutien et un encouragement en paroles, cultivaient des valeurs éducatives fortes, motivaient leurs enfants pour qu'ils ne ménagent pas leurs efforts et travaillent bien à l'école, apportaient un soutien tant direct qu'indirect aux devoirs, contribuaient à créer un environnement stimulant à la maison pour effectuer le travail scolaire et encourageaient les enfants à s'inscrire à des associations extrascolaires telles que les clubs de jeunes ou les activités proposées par l'église. Souvent les traditions et valeurs culturelles propres des parents conduisaient à des formes d'engagement particulières. Par exemple, une mère originaire de la République Dominicaine expliquait qu'elle répétait à ses enfants, comme l'avait fait sa propre mère, «l'école est sacrée, comme l'église».

En réponse à la seconde question, «quels sont les facteurs qui influencent l'engagement?", deux nouveaux thèmes sont apparus.

Les parents affirmaient que des facteurs "sociaux» et «scolaires» influençaient leur propre implication. Leurs expériences et leurs histoires de vie influençaient leur engagement. Parmi ces facteurs, on peut citer leur propre vécu éducatif à l'école, l'engagement de leurs parents lorsqu'ils étaient scolarisés, leurs représentations de l'engagement familial en fonction de leurs normes et de leurs valeurs culturelles, le poids de leurs autres responsabilités et leur disponibilité en temps.

L'étude permit de découvrir que des facteurs de nature relationnelle liés à l'école avaient un impact majeur sur la participation des parents. En engageant des relations bienveillantes et de confiance avec les parents considérés comme des partenaires du développement éducatif des enfants, l'équipe scolaire incitait les parents à s'engager et influençait la manière dont ils participaient au développement éducatif de leurs enfants. Les parents décrivaient le processus relationnel : en se focalisant en commun sur les enfants et leur apprentissage, la communauté scolaire avait su accueillir les parents dans l'école, apprécier leur participation et s'associer à eux.

Empruntant un concept qui appartient à la littérature sur les systèmes familiaux, ce «processus d'union» (Minuchin, 1981), c'est à dire d'accueil, d'estime et d'association avec les familles, crée une communauté scolaire dans laquelle chacun se sent «appartenir à une famille». Les parents répondent à cet 
état d'esprit en s'engageant dans des formes de participation qu'ils n'auraient jamais eux-mêmes envisagées et en devenant des membres fidèles de la communauté scolaire.

\section{LES TROIS COMPOSANTES DU PROCESSUS D'UNION}

\section{Accueillir}

Selon les parents, le processus d'accueil créait un sentiment d'appartenance réciproque entre l'école et les parents, qui les encourageait à être plus actifs dans la scolarisation de leurs enfants. A titre d'exemple, les parents estimaient que la communauté d'O'Hearn, c'est à dire le directeur, les enseignants, les autres membres de l'équipe et les parents, avait une façon accueillante de communiquer, qui manifestait leur désir sincère d'associer les parents à la vie de l'école. «A chaque rentrée, il est prévu que d'autres parents vous appellent et vous accueillent à l'école. C'est vraiment gentil. Ils organisent un petitdéjeuner pour les nouveaux parents. Je me suis arrangée pour y aller avec mon enfant qui hurlait. Il s'est senti vraiment bien là-bas et j'ai rencontré beaucoup de parents que je vois souvent maintenant. C'est ainsi que mon envie de voir comment chacun s'investissait a commencé. J'ai eu l'impression d'assister à une sorte d'“accueil au sein de l'école" et je me suis dit: "Regarde tous ces gens qui font tout cela pour les parents”. Alors j'essaie de faire ce que je peux chaque fois qu'ils donnent une fête, préparer de la nourriture ou autre chose. Je fais quelque chose pour dépanner. Cet événement m’a donné l'impulsion pour continuer et faire moi aussi ce que je peux pour l'école». Ou encore : "Si l'école n'était pas un lieu amical, il faudrait se forcer pour y participer. O'Hearn m'a donné l'occasion de m'engager. Je n'ai pas à me battre pour obtenir quoi que ce soit : tout est là à ma disposition pour que je sois tenu au courant».

Pour certains parents, l'environnement physique de l'école contribuait à leur sentiment d'être accueillis en franchissant l'enceinte de l'école. Les murs peints, décorés de nombreux exemples du travail artistique des enfants et la propreté de l'école participaient à ce sentiment d'accueil.

Ces propos des parents ont permis d'attirer notre attention sur un aspect important et souvent négligé du développement des relations école / familles. L'équipe de l'école oublie souvent les barrières qui existent entre les familles et l'école, à cause des différences de culture, de race et de milieu socio-économique ou parce que les parents sont intimidés par le personnel de l'école, voire en ont peur. L'équipe ne prend pas en considération le fait que l'environnement et la culture de leur établissement puissent ne pas inciter les familles à venir. Souvent, aucune attention n'est portée au développement d'initiatives systématiques d'accueil des familles au sein de la communauté scolaire. Les parents d'O'Hearn soulignaient l'importance du processus 
d'accueil pour la construction de relations positives entre les familles et l'équipe scolaire, son influence sur les raisons qui les incitent à participer à la communauté scolaire et les formes que prend cet engagement.

\section{Estimer}

Les parents se sentaient estimés par les membres de la communauté d'O'Hearn lorsque leurs efforts pour s'investir étaient validés et lorsqu'ils étaient traités par l'école comme de véritables partenaires du développement éducatif de leurs enfants. Encouragés par le directeur et les autres membres de l'équipe scolaire à participer à plusieurs comités de décision et à travailler sur des projets, ils étaient souvent associés à des parents bénévoles plus expérimentés. Cet encouragement et ce soutien les incitaient, dans certains cas, à s'investir de manière tout à fait inhabituelle pour eux.

Selon les parents, l'équipe de l'école était demandeuse d'informations sur l'apprentissage des enfants et en donnait à son tour. Ces échanges se passaient avec respect et bienveillance. Les observations des parents sur leurs enfants faisaient toujours l'objet d'écoute et d'attention de la part des enseignants. De nombreux parents disaient se sentir respectés et légitimés lorsque leurs idées et leurs préoccupations étaient entendues et prises au sérieux. Même si enseignants et parents n'étaient pas d'accord sur un sujet, la plupart des parents avaient le sentiment que les enseignants manifestaient bonne volonté, écoute, et désir de prendre en compte les informations qu'ils apportaient. De l'avis des parents interrogés, les relations avec l'équipe étaient réciproques dans la mesure où les idées et les propositions faisaient l'objet d'un partage et où l'on accordait une importance et un poids équivalents aux contributions des parents. "J'ai choisi l'école O'Hearn parce que selon moi c'était clairement la seule école publique où les parents étaient respectés et où enseignants, parents et enfants travaillaient vraiment ensemble de manière authentique». "O'Hearn demande aux parents de participer et sollicite des conseils en permanence. On dirait que l'école laisse les parents prendre les décisions, ce qui accroît l'engagement des parents. Les parents ont le sentiment de faire vraiment partie de l'école. Aux réunions, ça se passe exactement comme en famille. On discute, les parents disent ce qu'ils pensent être le mieux pour leurs enfants ou pour l'école dans son ensemble. Puis on passe au vote. Si vous avez des suggestions à faire, ils y sont toujours ouverts».

\section{S’associer}

Le personnel et les familles d'O'Hearn s'associent sur des thèmes éducatifs d'intérêt commun dont l'objectif est d'améliorer les chances d'apprentissage des enfants. D'après les parents, c'était la priorité donnée par l'école à un enseignement et un apprentissage toujours meilleurs, pour tous les enfants, qui leur donnait le sentiment d'être associés à la communauté scolaire. 
L'ensemble des activités de participation des familles, des pièces de théâtre aux journées portes ouvertes ont contribué à améliorer les résultats de tous les enfants.

Selon les parents, le personnel d'O'Hearn se souciait des enfants et avait pour eux de grandes attentes. Grâce à cet intérêt de l'équipe pour l'éducation et pour le bien-être global des enfants, les parents et le personnel de l'école se retrouvaient sur un terrain commun. "Ils m'ont donné l'impression qu'ils étaient là pour instruire mon enfant. Ils me donnaient toujours des détails pertinents à son sujet. Je savais qu'ils prêtaient attention à mon fils, qu'ils connaissaient ses forces et ses faiblesses. Cela me faisait du bien. Ils me disaient des choses sur lui que j'aurais pu leur dire, avant même que les mots ne sortent de ma bouche. Je me disais : "Ils connaissent mon fils" ". "J'ai trois enfants inscrits à l'école O'Hearn. La proximité que semblent avoir les enseignants avec mes enfants a pour résultat de me donner envie d'être plus investi dans leur vie. Les enseignants semblent plus concernés, plus engagés dans l'éducation des enfants. Cela donne envie d'être soi-même plus actif dans la vie de son enfant lorsqu'il rentre à l'école».

Ce processus d'union, qui place l'éducation des enfants au centre, rapproche parents et équipe scolaire. Les deux parties se rallient à une cause commune qui a du sens et de l'importance pour tous deux. Les parents plaident avec ferveur en faveur de l'école lorsque le personnel manifeste son intérêt et son engagement à l'égard de l'éducation de leurs enfants. En collaborant d'égal à égal à l'éducation des enfants, parents et enseignants construisent en partenariat une relation de confiance et de compréhension. Cette attention centrée sur les enfants est ce qui relie les parents, les maintient engagés et nourrit leur sentiment d'être des membres importants de la communauté scolaire.

\section{Conséquences pratiques Dissiper un mythe}

Les témoignages des parents d'O'Hearn indiquent que le personnel scolaire ne devrait pas supposer a priori que les parents qui ont un bagage ethnique et/ou viennent d'un milieu socio-économique différents se soucient moins de l'éducation de leurs enfants, font preuve de passivité à cet égard ou encore ne comprennent guère l'importance de leur participation à l'éducation de leurs enfants. Les écoles où a cours cet a priori risquent d'éprouver des difficultés à créer un environnement favorable au développement de partenariats efficaces entre les parents et l'école.

\section{Reconnaître plusieurs formes d'engagement familial}

L'étude montre que l'engagement des parents dans l'éducation des enfants peut prendre des formes non reconnues par les écoles qui ont une vision 
étroite de ce qui constitue une participation légitime. Certaines écoles n'accordent de légitimité qu'aux activités sanctionnées et dirigées par elles. Elles limitent ainsi la façon dont les parents peuvent s'investir et négligent les autres formes d'engagement des familles de milieux et de cultures différents. En ne reconnaissant pas la diversité des formes de participation et en ne fournissant pas une pluralité de choix à la participation des familles, les écoles restreignent inconsciemment les formes que peut recouvrir la participation des parents à la scolarité de leurs enfants. Les résultats de l'étude laissent entendre que c'est l'insuffisance d'options, plutôt que le manque d'intérêt pour l'engagement, qui est un facteur restrictif de la participation parentale.

\section{Comprendre le processus d'implication}

S’il faut ne citer qu'une découverte mise à jour par les témoignages des parents d'O'Hearn, c'est que les facteurs liés à l'école ont une influence primordiale dans l'engagement parental. Le processus d'union avec les familles crée un environnement qui permet à de nombreux parents de dépasser leurs expériences antérieures négatives de l'école et/ou leur sentiment d'aliénation. Voyant leurs initiatives validées, les parents sentent qu'ils contribuent de façon significative à la communauté scolaire, ce qui a pour effet d'accroître leur efficacité et leur confiance en leur capacité à aider leurs enfants. S'associer aux familles en s'axant sur les enfants et leur environnement d'apprentissage a rassemblé les dix-huit parents et l'équipe scolaire autour de buts et d'objectifs communs.

Ces découvertes suggèrent que chacun des trois éléments constitutifs du processus d'union - accueil, estime et association - encourage une participation active à la maison et à l'école et développe des relations de partenariat entre parents et équipe scolaire. Enfin, le processus d'union a pour effet de renforcer l'aptitude des parents à aider leurs enfants et améliore ainsi la capacité éducative globale de l'école.

Même dans une communauté scolaire bienveillante, des conflits et des tensions surgissent entre les parents et l'équipe de l'école. Certains parents affirment ne pas toujours être d'accord avec le style de gestion participative du directeur Henderson et disent avoir des discussions et des désaccords avec lui. D'autres se souviennent d'avoir été en colère contre l'équipe scolaire. Cependant, la confiance sur laquelle reposent les relations entre les familles et l'équipe crée une communauté où les conflits se produisent mais sont souvent résolus.

Cette étude suggère que l'engagement des parents dans l'éducation de leurs enfants est influencé par un certain type de culture scolaire qui valorise les relations avec les parents et travaille très activement à établir des relations respectueuses et réciproques avec eux. Les propos employés par les dix-huit parents interrogés pour l'étude insistent sur les facteurs relationnels et la confiance comme influençant beaucoup leur participation. De nombreux 
programmes scolaires, pourtant, mettent l'accent sur l'engagement familial et non sur le processus consistant à développer les liens entre les familles et l'équipe scolaire.

Ces découvertes suggèrent aussi que l'engagement, le soutien et la participation active du directeur sont nécessaires à la création et au maintien d'une culture scolaire qui englobe une participation totale des familles. De l'avis des parents et des membres de l'équipe interrogés, l'un des éléments qui a le plus influencé la participation des familles a été l'exemple donné par le directeur. Dès sa prise de fonction, William Henderson a préconisé un haut niveau de participation familiale et a manifesté son engagement à travers ses propres actions. Il a également insisté auprès de l'équipe scolaire sur l'importance de l'investissement des familles. Cette attitude a eu pour effet de développer une communauté scolaire dont les membres étaient «sur la même longueur d'ondes» en ce qui concerne l'engagement des parents dans la scolarité de leurs enfants.

Trop souvent, les écoles n'accordent de l'importance à l'engagement des familles qu'en paroles et leurs tentatives pour inciter les parents à être actifs sont timides. Certaines écoles engagent un parent isolé ou une seule famille pour être coordinateur et organiser entièrement la participation des familles. Sans le soutien et l'engagement du directeur et de l'équipe scolaire, des programmes de cette nature se désintègrent bien souvent. Alors que l'échec de ces programmes est souvent imputé au manque d'intérêt des familles, l'étude montre que le soutien apporté par le responsable de l'école et par l'équipe sont l'élément crucial qui garantit le succès des initiatives en faveur de l'engagement familial.

\section{Pour continuer LA ReChERCHE}

On sait qu'au fur et à mesure que les enfants grandissent, la participation des familles à leur scolarité faiblit (Henderson \& Berla, 1994). Cette étude, qui a été menée au sein d'une école élémentaire, ne permet pas de savoir si les facteurs identifiés par les dix-huit familles d'O'Hearn comme influençant leur engagement s'appliquent également aux expériences des parents des middle ${ }^{10}$ et des high schools ${ }^{11}$. Les mêmes facteurs sociaux et scolaires influencent-ils leur participation? Pour le savoir, il faudra mener des études approfondies au sein de middle schools et de high schools.

La présente étude a montré que le soutien et l'engagement du directeur sont des facteurs déterminants pour la création d'un environnement et d'une culture scolaires favorisant la participation familiale. Quel est le type de direction scolaire qui encourage le développement d'une communauté scolaire

10. Les middle schools accueillent les enfants entre 5 et 13 ans.

11. Les high schools accueillent les adolescents entre 14 et 18 ans. 
engagée? De quelles compétences doit faire preuve le directeur pour construire des partenariats efficaces entre l'école et les familles? De plus amples recherches sont nécessaires.

Et que doivent savoir les enseignants pour favoriser les partenariats avec les familles? Il faut le savoir si l'on veut concevoir des programmes en formation initiale et en formation continue pour les enseignants et les administrateurs qui permettent de développer les compétences nécessaires au développement de partenariats avec les familles.

Cette étude n'a pas cherché à identifier les types de relations existant entre les membres de l'équipe enseignante d'O'Hearn. Cependant, il est intéressant de remarquer qu'à l'école O'Hearn, une culture de partenariat et de collaboration existe au sein de l'équipe enseignante. Les cours sont co-animés par deux professeurs qui travaillent en étroite collaboration ${ }^{12}$.

Une telle culture favorisant le travail d'équipe entre les enseignants d'O'Hearn influence t-elle alors la dynamique de partenariat avec les familles? Les écoles n'ayant aucune histoire ou culture de collaboration et de partenariat à l'intérieur des membres de son équipe peuvent-elles établir des partenariats efficaces avec les familles? D'instinct, on serait tenté de répondre par la négative. Cela pose en tout cas une question intéressante qui mériterait de nouvelles recherches.

Les dix-huit parents d'O'Hearn interrogés pour cette étude veulent que leurs enfants réussissent à l'école; ils comprennent l'importance de la participation de la famille à l'éducation de leurs enfants. En outre, ils affirment que les facteurs sociaux et scolaires influencent leur participation. Ils révèlent également que leur engagement est influencé de manière significative par un environnement scolaire qui «fait penser à la famille».

Les limites de cette étude, son centrage sur les parents d'une seule école et la taille de l'échantillon (dix-huit parents et sept membres du personnel scolaire), rendent impossible la généralisation des découvertes au-delà du cadre de la recherche. Néanmoins, les résultats apportent des renseignements pertinents sur la participation des familles dans les écoles urbaines. Cette étude montre que le personnel de l'école doit soutenir une culture familiale à l'école, où tous les membres de la communauté scolaire sont respectés et estimés. L'étude fait la démonstration que des relations respectueuses, où le pouvoir est partagé entre le personnel de l'école et les membres des familles, fournissent le ciment qui maintient la cohésion de la communauté et influence l'engagement des parents. 


\section{RÉFÉRENCES BIBLIOGRAPHIQUES}

DAVIES D. (1988): Low-income parents and the schools : A research report and a plan for action. Equity and Choice, 4 (3), 51-57.

EPSTEIN J.L. (1988): How do we improve programs for parent involvement? Educational Horizons, 66 (2), 58-59.

EPSTEIN J.L. (1991): Effects on student achievement of teachers' practices of parental involvement. Advances in reading/language research (Vol. 5, pp. 261-276). Greenwich, CT : JAI Press.

EPSTEIN J.L. (1995): School/family/community partnerships : Caring for the children we share. Phi Delta Kappan, 76 (9), 701-712.

HENDERSON A., \& BERLA N. (1994): A new generation of evidence: The family is critical to student achievement. National Committee for Citizens in Education. 